\title{
Shaping the spectral characteristics of fiber Bragg gratings written in optical fiber taper using a phase mask method
}

\author{
Tomasz Osuch, ${ }^{* 1,2}$ Kazimierz Jędrzejewski, ${ }^{2}$ Lech Lewandowski, ${ }^{2}$ and Wiesław Jasiewicz ${ }^{2}$ \\ ${ }^{1}$ Instytute of Electronic Systems, Warsaw University of Technology, Nowowiejska 15/19, 00-665 Warsaw, Poland \\ ${ }^{2}$ National Institute of Telecommunications, Szachowa 1, 04-894 Warsaw, Poland
}

Received October 26, 2012; accepted December 22, 2012; published December 31, 2012

\begin{abstract}
Fabrication of fiber Bragg gratings using a phase mask method, despite the substantial advantages, has a significant drawback. In this technique, the phase mask period determines the Bragg wavelength, and thus limits the possibilities of shaping its spectral characteristics. The paper presents the possibility of modifying the transmission and reflection spectra of fiber Bragg gratings written in a thermally tapered optical fiber using complex UV irradiation through a uniform phase mask. Structures with two Bragg wavelengths and with a narrow transmission band in the spectral characteristics were achieved by modifying the amplitude of refractive index changes and effective index distribution along the fiber taper.
\end{abstract}

In a commonly used phase mask technique for fiber Bragg grating (FBG) fabrication the Bragg wavelength $\lambda_{B}$ depends on an effective refractive index of fiber core $n_{\text {eff }}$ and phase mask pitch $\Lambda_{\mathrm{PM}}$, according to the following formula $[1,2]$

$$
\lambda_{\mathrm{B}}=n_{e f f} \cdot \Lambda_{P M}
$$

Thermal tapering of optical fiber results in a change of core and cladding diameters, which causes an effective refractive index variation along the fiber axis [3]. Assuming a perfect conical shape of the optical taper region, the dependence of an effective refractive index for $\mathrm{LP}_{01}$ mode in a single-mode optical fiber on its diameter is shown in Fig. 1.

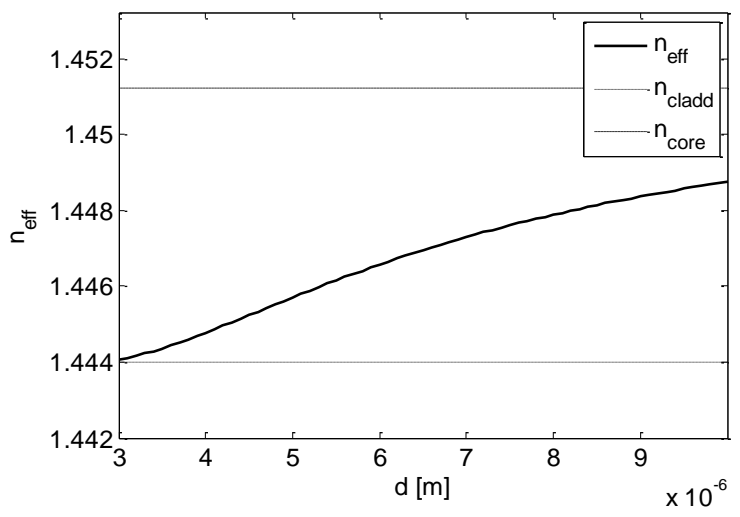

Fig. 1. Effective refractive index for $\mathrm{LP}_{01}$ mode as a function of optical fiber diameter.

\footnotetext{
*E-mail: T.Osuch@elka.pw.edu.pl
}

The characteristic shown in Fig. 1 was calculated assuming the following parameters of the optical fiber: refractive index of cladding $\mathrm{n}_{\text {cladd }}=1.4440$ and core $\mathrm{n}_{\mathrm{co}}=1.4512$, wavelength $\lambda=1536 \mathrm{~nm}$. Thus, the variation of an effective refractive index on optical fiber diameter can be utilized for writing complex Bragg structures through a uniform phase mask. Therefore, Eq. (1) takes the form:

$$
\lambda_{\mathrm{B}}(x)=n_{e f f}(x) \cdot \Lambda_{P M},
$$

where: $x$ is the direction along the fiber axis. Eq. (2) and the characteristic from Fig. 1 show that depending on the Bragg grating location in the taper region of an optical fiber it is possible to obtain any Bragg wavelength within the range of few $\mathrm{nm}$ in the third telecommunication window.

In the first step standard germanium doped telecommunication single mode optical fiber was thermally tapered using a gravitational method. Using this technique, 20-35mm length asymmetric fiber-optic tapers were achieved. However, in this case the differences in lengths of taper regions were irrelevant, because fiber Bragg gratings were written in one of them. Then the tapered fibers were hydrogen-loaded at 120bars in room temperature for two weeks to enhance their photosensitivity. Fiber Bragg gratings were written by a scanning uniform phase mask with $1061 \mathrm{~nm}$ pitch using a frequency doubled CW argon ion laser with output optical power $100 \mathrm{~mW}$ [4]. In this technique, scanning enables to produce fiber Bragg gratings of various length, while the total length of gratings is limited only by phase mask dimensions and/or the length of the taper region.

Due to the difference in fiber diameter along the fiber axis in the tapered region, and thus non-uniform concentration of hydrogen and germanium in a fiber core, the dynamic of fiber Bragg grating growth varies. In this case, the UV exposure doses were dynamically controlled during FBG writing, and were significantly greater in the areas of a small diameter.

The setup for fiber Bragg grating fabrication in fiber-optic tapers using a phase mask method and with direct control 
of spectral transmission/reflectance characteristics is shown in Fig. 2.

Strictly specified areas of taper were illuminated by a UV laser source through a uniform phase mask PM. The size of the arrows in Fig. 2 corresponds to UV radiation doses, which were significantly greater in in the region of FBG1. The exposures of particular areas of optical tapers were realized by a moving phase mask and optical fiber with respect to the UV laser beam. The FBGs writing process was monitored by spectral transmission or reflectance characteristics depending on the connection of optical fiber with a measuring system. The spectral characteristics were measured using a broadband source BBS (superluminescent diode with the spectral range c.a. 1500$1560 \mathrm{~nm})$ and an optical spectrum analyzer OSA. For reflectance spectrum measurement an optical circulator OC was used additionally.

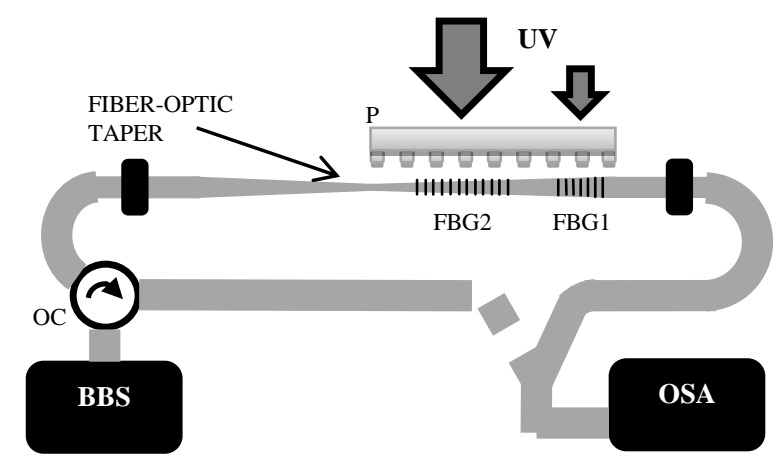

Fig. 2. Setup for fiber Bragg grating fabrication in fiber-optic tapers using phase mask method, FBG1 and FBG2 - fiber Bragg gratings, BBS - broadband source, OSA - optical spectrum analyzer, PM - phase mask, OC-optical circulator.

Using tapered optical fiber, complex structures were fabricated, consisting of two fiber Bragg gratings separated by a few $\mathrm{mm}$. The lengths of the tapered regions (with Bragg gratings) were $15-20 \mathrm{~mm}$ while the waist regions had $15-40 \mu \mathrm{m}$ in diameter. Inscription conditions were optimized for obtaining two Bragg minima in the spectral transmission characteristic in the first case and a narrow transmission band in the reflected spectrum in the other.

The spectral transmission characteristic of the first structure is shown in Fig. 3, where two Bragg minima are noticeable. The first minimum, at the long wavelength side, with a Bragg wavelength of $1536.33 \mathrm{~nm}$ has a spectral width narrower than the second one. This is because the fiber Bragg grating (FBG1) was written in the region with a small change of optical fiber diameter, and thus minor effective refractive index variations. For comparison, the Bragg wavelength of a uniform grating written in non-tapered optical fiber using the same phase mask (with $1061 \mathrm{~nm}$ pitch) and a similar UV exposure dose has the Bragg wavelength c.a. 1536.50nm.

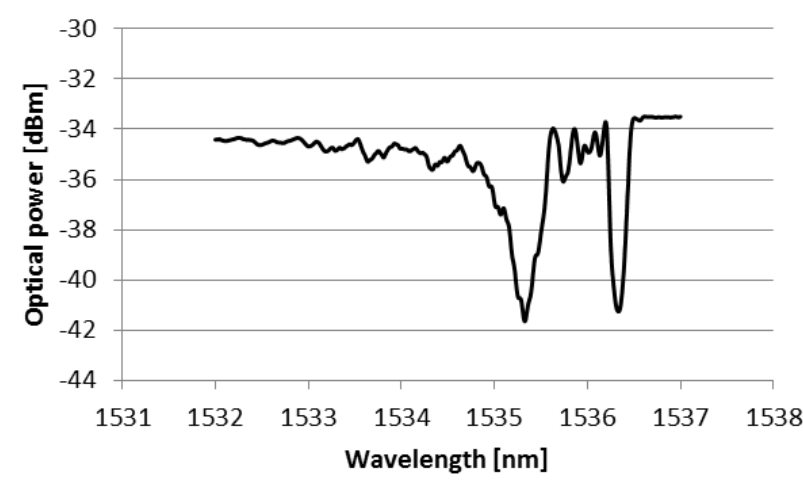

Fig. 3. Spectral (transmission) characteristic of two fiber Bragg gratings written in taper region and separated $5 \mathrm{~mm}$.

The second Bragg minimum with a central wavelength of $1535.33 \mathrm{~nm}$ has a significantly wider spectral width than the first one, although both Bragg gratings have the same length. The reason is that the second grating FBG2 was written on the FBG2 is written in the region of taper with a considerable variation of diameter, and thus an effective refractive index. In addition, several times longer exposure time (in comparison with FBG1 writing) made changes in the refractive index, and thus broadened spectral characteristics on the short wavelength side could be observed. The values of the parameters of the two Bragg minima in the spectral transmission characteristics, which correspond to FBG1 and FBG2 were summarized in Table 1. Here the spectral width is defined at $3 \mathrm{~dB}$ above the minimal optical power level (corresponding to the Bragg wavelength).

Table.1. Summary of the parameters of the Bragg minima in the spectral transmission characteristic shown in Fig. 3.

\begin{tabular}{|l|c|c|}
\hline Parameter & FBG1 & FBG2 \\
\hline Bragg wavelength [nm] & 1536.33 & 1535.33 \\
\hline Spectral width [nm] & 0.17 & 0.30 \\
\hline Depth [dB] & $\sim 7.5$ & $\sim 7.0$ \\
\hline
\end{tabular}

The second structure consists of two $6 \mathrm{~mm}$ long fiber Bragg gratings separated by $\sim 1 \mathrm{~mm}$. The first section (FBG1) was written in the tapered region with a fiber diameter of $90-110 \mu \mathrm{m}$ by scanning the phase mask at a time of $180 \mathrm{sec}$. In the case of the second section (FBG2), the exposure time was increased to $750 \mathrm{sec}$. in order to obtain similar reflectance. As a result, the Bragg structure with a very narrow transmission line in the spectral reflectance characteristic was achieved (Fig. 4). The center wavelength of the transmission gap (dip in characteristic in Fig. 4) is $1533.81 \mathrm{~nm}$, while its spectral width is $\sim 30 \mathrm{pm}$. In this case, the spectral width is defined at the power level $3 \mathrm{~dB}$ above the optical power level which corresponds to the gap wavelength. 


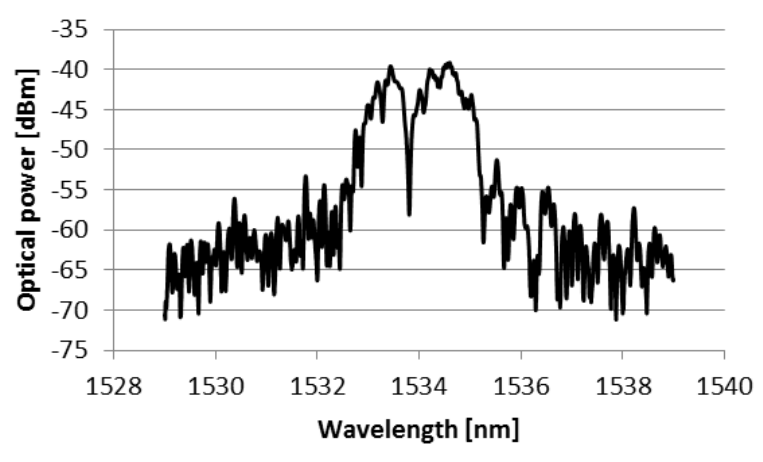

Fig.4. Spectral (reflectance) characteristic of Bragg structures with a narrow transmission band.

The values of transmission gap parameters in the spectral reflectance characteristic of the second presented structure are shown in Table 2.

Table.2. Summary of the main parameters of a narrow transmission gap in the spectral reflectance characteristic shown in Fig. 4.

\begin{tabular}{|l|c|}
\hline Parameter & Value \\
\hline Gap wavelength [nm] & 1533.81 \\
\hline Spectral width [nm] & 0.03 \\
\hline Depth [dB] & $\sim 18$ \\
\hline
\end{tabular}

The presented method of complex inscription of fiber Bragg grating in tapered optical fibers allow for shaping and controlling their spectral characteristics. An appropriate choice of FBGs locations in optical taper and UV exposure time control allows to obtain fiber Bragg grating based passive optical components with complex spectra, such as: structure with two minima in its spectral transmission characteristic as well as with narrow transmission gap of the range of tens of pm).
The first structure can be used as a temperature and/or a strain sensor. While the temperature responses of both gratings are similar, the influence of strain on their spectral characteristics significantly differs due to their different taper profiles and diameters (and thus elongation) in places where FBGs were written. The second structure, with a narrow transmission band, may be utilized as a highly selective transmission band-pass filter as well as a sensing element. The repeatability of fabrication of such Bragg grating structures significantly depends on the following factors: taper profiles, precision of FBGs writing in the appropriate places of the fused taper as well as exact determination of the width of a UV scanning beam (and therefore actual length of FBGs). Similar taper profiles are easily obtained in a horizontal method rather than a gravitational one [5]. The UV beam size and thus FBGs length can be also relatively simply determined with sufficient, sub-milimeter precision. Additionally, in order to achieve satisfactory reproducibility, the growth of the spectral characteristic of a written FBG structure is monitored during exposure and therefore dynamical control of a fabrication process is provided.

This work was supported by National Science Centre under the Project NCN 4707/B/T02/2011/40.

\section{References}

[1] A. Othonos, K. Kalli, Fiber Bragg gratings: fundamentals and applications in telecommunications and sensing (Norwood, Artech House 1989).

[2] R. Kashyap, Fiber Bragg gratings (Boston, Academic Press 1999).

[3] K.C. Byron, K. Sugden, T. Bricheno and Bennion, Electr. Lett. 29, 1659 (1993)

[4] T. Osuch, P. Gąsior, L. Lewandowski, Proc. SPIE 5775, 222 (2005).

[5] K. Jędrzejewski, Opto-Electr. Rev., 8, 153 (2000). 